\title{
Bindung durch Pfannkuchen? Das Volkskonzept in Mel'nikov- Pečerskijs „V lesach“
}

\begin{abstract}
Welche domenvolle Untersuchung hat nicht das Sein den Philosophen verursacht! Ist es eines oder vieles, eins mit dem Denker oder verschieden von dem Nichts des Gedankens? Unnutze Fragen! Das Sein ist eins mit dem Essen; sein heißt essen; was ist, isst und wird gegessen." (Feuerbach 1971, 358)
\end{abstract}

Summary

Nations do not seem to be united primarily by eating and drinking. Mel'nikov- Pecherskijs "V lesach", however, shows an opposite effort. Mel'nikov understands narod as a prospering Russian nation, not - like many of his contemporaries - as the downtrodden masses, the peasantry. The characters in "V lesach" are tradesmen and rich. Mel'nikov links his economic vision - the building-up of small manufactories - with the variety of dishes his heroes serve. Food becomes a symbol of softened economic advance. Yet in one way the author sticks exclusively to an older patriarchal world. While food unifies the classes, especially tradesmen and peasants, it shows a significant difference between the sexes. Mel'nikov depicts a feudalist as well as a nationalist society, the contradiction of which can be demonstrated by his use of pancakes.

Ludwig Feuerbach reflektiert 1850 die neuesten Entdeckungen der Chemie, darunter ein Buch von Jakob Moleschott mit dem Titel „Lehre der Nahrungsmittel. Für das Volk“. Der Unterricht in Chemie, v. a. die Information über einen Stoffwechsel gibt Feuerbach die entscheidenden Argumente gegen die vermeintliche Schöpfungstätigkeit des menschlichen Geistes. Der Geist, das Lieblingskind idealistischer Philosophen, zeigt sich aus der neuen Perspektive als bloßes Anhängsel des Magens. Er produziert gar nichts, sondern ist Effekt des Futters, das man ihm zuführt. Kurz und knapp: „wo kein Fett, da ist auch kein Hirn" (Feuerbach 1971, 358). Feuerbach verlacht nicht nur die herkömmliche Philosophie, wenn er Essen und Trinken als ,Wesen der Wesen' bestimmt, seine materialistische Erkenntnis zieht politische Forderungen nach sich. Sowohl der Intellekt des Individuums, als auch der geistige Zustand einer Gesellschaft hängen von der Nahrung ab, und damit stellt sich die Frage nach einer gerechteren Verteilung der Lebensmittel. Diese handfeste Argumentation verdankt sich einem ebenso handfesten Hintergrund. Feuerbach und Moleschott haben die irische Kartoffelfäule gut im Gedächtnis, der 1845 mehrere Millionen Menschen zum Opfer fallen. Zum Zeichen tödlicher Abhängigkeit avanciert, verbietet quasi die Kartoffel selbst jede Spekulation über einen umgekehrten Einfluss der Seele auf den Körper. Alle moralischen Lehren sind nach Feuerbach überflüssig, will man das Volk bessern, so gebe man ihm „,statt Deklamationen gegen die Sünde bessere Speisen“ (ebenda, 367). Sowohl Bildung als auch Gesinnung der Menschen sind abhängig von ihrer Nahrung.

Die materielle Basis des Subjekts wird auch dann philosophisch relevant, wenn es um eine Kritik dieses Subjekts geht. Nicht nur die Konstruktion des Daseins, sondern auch seine postmoderne Dekonstruktion setzt beim Essen an. So hinterfragt Jacques Derrida nach- 
drücklich den Zusammenhang von Identität und Nahrungsaufnahme, ja er legt diese Verstrickung erneut bloß (Derrida 1998, 267-298). Die unterschiedlichen Ziele von Materialismus und poststrukturalistischer Philosophie seien freilich unbestritten. Schon die Kardinalfrage aller Nahrungsapostel - Fleisch oder nicht? - wird verschieden beantwortet. Während Feuerbach den Fleischverzehr befürwortet und damit dem sozialen Gefälle Abhilfe schaffen will, kritisiert Derrida die subtile Vormachtstellung des Menschen unter allem Lebendigen. Die selbstherrliche Voraussetzung des Subjekts zeigt sich im Töten von Tieren, ein Töten, das kein Mord sein soll und vom Mord - an Menschen - fein säuberlich unterschieden wird (ebenda, 289). Damit verbindet sich zwar kein Plädoyer für vegetarische Kost, die carnivore Gesellschaft kommt bei Derrida dennoch schlechter weg, da sie zumeist auch phallogozentrisch organisiert ist. Fleischessen, ein Instrument der Macht über andere Lebewesen, geht einher mit männlicher Dominanz. ${ }^{1}$ Dem Machtverhältnis zwischen den Geschlechtern wird auch in „V lesach" nachzuspüren sein. Zunächst und grundsätzlich trifft auf Mel'nikovs Werk aber die materialistische These zu: Geist, Seele und Identität des Menschen hängen von seinem Essen ab, oder - um mit Feuerbach zu sprechen: Die Frage nach dem Ich wird vom aufgerissenen Mund gelöst (Feuerbach 1971, 359) und die Antwort ergibt sich aus dem, was man sich in den Rachen stopft.

Über die Konstruktion kollektiver Identitäten ist damit noch nichts ausgesagt, die Einverleibung von Lebensmitteln findet jedoch kaum beliebig, spontan oder gar nur individuell statt. Sie folgt bekanntermaßen Regeln, Menschen essen und hungern sogar miteinander. Nahrung bindet, aber warum soll sie Völker binden? Der Begriff des Volkes hat darüber hinaus mehrere Bedeutungen. Im russischen Reich sind zwischen 1850 und $1880^{2}$ besonders zwei relevant: das Volk im Sinne von Nation, das russische Volk also und das Volk im Sinne der Unterschicht, народ als Bauern. ${ }^{3}$ Mel'nikov, das sei hier vorweggeschickt, akzentuiert entschieden die erste Größe und kommt v. a. ohne Widerspruch zwischen dem Russischen und gesellschaftlichen Klassen aus.

Generell definieren sich Nationen selten über Nahrung. Das deutlichste Beispiel bietet das jüdische Volk, dessen Gemeinschaft stark auf Essensregeln beruht. Studien, die sich der Verbindung von Nation und Nahrung widmen, beziehen sich deshalb vorrangig auf die Juden, wobei Deutungen und Begründungen der mosaischen Diätgesetze weit auseinandergehen. ${ }^{4}$ Der jüdischen Konzentration des Volkes antwortet als Gegenbewegung - so sieht es der semiotische Teil der Forschung - die Mission des Christentums, das nun um-

1 „Die virile Kraft des erwachsenen Mannes, Vaters, Gemahls oder Bruders [...] gehort dem Schema an, das den Subjektbegriff beherrscht. [...] wer hätte in unserer Gegend eine Chance, Staatsoberhaupt zu werden [...], der offentlich, also beispielhaft erklärte, Vegetarier zu sein? Der Chef muss Fleischesser sein" (Derrida 1998, 292).

2 In diese Zeitspanne fallen erzahlte Handlung und Textentstehung.

3 Hinzu kommen die von offizieller Seite propagierte Volkstumlichkeit (народность), die sich als autoritär verordneter Staatspatriotismus beschreiben lässt und der Mythos vom heiligen Russland, der auf den Pfeilern Land, Glaube, Zar ruht. Die verschiedenen Varianten des Volksbegriffes in Russland diskutieren Hobsbawm 1991, 62ff., 102ff.; Hosking 1990 und 1997; Kappeler 1990 und 1992, 198ff.; Knight 2000.

4 Sie variieren von semiotischen, formalen Erklärungen bis hin zu pragmatisch-ökologischen, das Schweinefleischverbot erscheint zum einen als reine Demarkationslinie, der territorialen Integrităt des Staates Israel ăquivalent (Douglas 1997, 51), oder geht zum anderen auf den Mangel an Wald zurluck: Mit der bloßen Hitze kommen Schweine einfach nicht zurecht (Harris 1997, 71). 
gekehrt durch Freiheit von Regeln markiert ist (Soler 1997, 65). Hier erst kommt der Mensch als Allesfresser zum Vorschein. Unreine Speisen gibt es nicht mehr, einschränkend wirkt jedoch die temporale Abstufung. Zu bestimmten Zeiten darf man nicht alles essen, und die russisch-orthodoxe Kirche geht hier - wie viele Reiseberichte bezeugen strenger als ihre westeuropäischen Schwesterkirchen vor. Allein die Unterscheidung von Fastentagen - deren mindestens $180 \mathrm{im}$ Jahr zu verzeichnen sind ${ }^{5}$ - und Feiertagen, hat weitreichende Auswirkungen auf die Ernährung der Bevölkerung, auf ihre Gesinnung und ihr Kollektiv. Ein nationaler, von der Religion inszenierter Zusammenhalt wäre deshalb auch in Russland denkbar. Um dem Verbund anzugehören, sollte man zumindest die Fastenzeiten respektieren. Reflexe dieser Vorschrift finden sich in Mel'nikovs „V lesach“, jedoch geht der Autor humorvoll damit um: Ein deutscher Arzt etwa, der die Nonne Manefa kurieren soll, unterliegt der Kritik des Kutschers Dementij. Der Arzt isst nicht richtig, er fastet nicht und fält damit aus der Kategorie der Russen, ja sogar des Menschlichen heraus. Wie ein Hund, so Dementij, fresse der Mann verbotene Speisen. Für den Kutscher erübrigt sich jede weitere Identifikation des sündigen Gegners: „Да лекарь-от из немцев аль бусурманин-какой...“ (III, 77) ${ }^{6}$ Die Sache ist Dementij gleich, ob Christ oder NichtChrist: falsch bleibt falsch.

Das harsche Urteil des Kutschers wirkt jedoch komisch, weil er an gleicher Textstelle einen Wettbewerb zwischen Kirche und Essen inszeniert. Dem Moskauer Gesandten der Altgläubigen entgegnet er zur Verteidigung seiner Heimat: Bei euch läuten mehr Glocken, bei uns gibt es mehr zu essen. Und trotz aller Fasten lautet Dementijs Resümee: „У нас по лесам житье-то, видно, приглядней московского будет [...]. По крайности ешь без меры, кусков не считают.“ (III, 79)

Die Verbindung von Essen und Religion scheint in „V lesach“ labil, lockerer als man es in einem ,Roman' vermuten würde', dessen Helden allesamt den Raskol'niki angehören. Zwar bedient sich die Differenzierung von Russischem und Ausländischem bisweilen der Motivik des Essens, der religiöse Bezug aber geht verloren. National ist offenbar nur noch die Küche, auch ohne Kirche. Mel'nikovs Figuren wissen um Schleichwege, auf denen man sich mit Fisch und Portwein sogar während der vorösterlichen Fastenzeit (Великий пост) vergnügen kann: Wer auf Reisen ist, darf bewirtet werden'; die Zu-HauseGebliebenen nutzen die Gelegenheit, sie schließen sich ihren Gästen bereitwillig an (II, 267), und der Rum, dessen euphemistische Umschreibung nun „Fastenmilch“ lautet, fließt in Mengen (II, 270).

5 Frierson 1997, 56, und Heretz 1997, 69, gehen von 180 Fastentagen aus, Smith; Christian 1984, 10, von 192 bis 216 Tagen. Die Zahl der Fastentage variiert in Abhängigkeit vom Osterdatum, das seinerseits die Zeitspanne zwischen Pfingsten und Peter und Paul bestimmt, in der, lange oder kurz, gefastet wird.

Die folgenden Zitate beziehen sich auf Mel'nikov 1976, Bde. 2-4. Die Bandnummern sind in römischen, die Seitenzahlen in arabischen Ziffern angegeben.

$7 \quad$ „V lesach" kann gattungsgeschichtlich auch als spăter Ableger des Epos eingestuft werden. Levin 1958, 3 spricht von einer „Epopöe“ und zitiert Gorkijs Erzăhlung „V ljudjach“, in der von einem „ruhmreichen russischen Poem" die Rede ist (ebenda, 28). Da der Roman die wohl heterogenste epische Gattung darstellt, unter die sich im Zweifelsfalle auch noch „V lesach“ subsumieren lassst, werde ich im Folgenden an dieser (Hilfs-)Bezeichnung festhalten. Von "transzendentaler Obdachlosigkeit" (Lukács) ist ,in den Wäldern“ allerdings nichts zu spuren.

Diese Regel gilt auch fur andere Religionen, so etwa fur den Islam und die katholische Kirche. 
Die entscheidende Manipulation zugunsten des Feierns aber übernimmt der Verfasser selbst: Er zentriert durch permanente Sprünge die Handlung um immer gleiche Festtage. Der Roman spielt zwischen Januar und Juli eines Jahres (es handelt sich vermutlich um das Jahr 1852), , er schließt damit die wichtigste Fastenzeit des Jahres ein, der Leser erfährt aber mehr und mehrfach von Johannes- und Petersfest. (III, 59, $201 \mathrm{ff}$.; IV, 87ff., 157, $177 \mathrm{ff}$. $)^{10}$. Die russischen Menschen hinter der Wolga leben gut - dieser Schluss ist vom Autor vorprogrammiert. Fasten heißt ,in den Wäldern“ keineswegs Entbehrungen erleiden, und wenn schon dem Ausland getrotzt werden soll, so entscheidet die Sache der Bauch, nicht die Religion.

Auch topographisch gesehen begibt sich Mel'nikov in den Bauch des russischen Reiches. Die Ereignisse spielen am linken Wolgaufer, nahe Nižnij Novgorod, in einem Gebiet, das schon zu Beginn des Textes als „старая Pусь“ als ursprünglich, echt und ohne den Zusatz des Ausländischen beschrieben wird (II, 8-11). Die Hauptfigur des Romans ist der Kaufmann Patap Maksimyč Čapurin. Er betreibt eine Drechslerei und fungiert als Arbeitgeber für 20 umliegende Dörfer (II, 47). Sowohl in gesellschaftlicher als auch in familiärer Hinsicht erfültt er die Rolle des Ernährers. Seine Frau Aksin'ja spricht ihn devot mit „кормилец“ an (II, 25), Patap veranstaltet sog. кормы für die Bauern, er lädt sie zu Festessen ein, bei denen das Volk überaus üppig tafelt. Daneben unterstützt er mit Geld und Lebensmitteln seine Schwester Manefa, die als игуменья einem altgläubigen обитель vorsteht. Die Nahrungskette setzt sich fort: Das Kloster speist seinerseits die ortsansässigen Bauern, die mildtätigen Aktionen rufen den Vergleich mit einem „гостиный двор“ auf den Plan (IV, 142), dessen doppelte Funktion als Gäste- und Handelshaus hier mitgedacht ist. Im Speisen konkurrieren die Altgläubigen mit den Nikoneanern, sie halten ihre Schäfchen durch Essen, nicht durch Dogmen zusammen und die offizielle Kirche hat in diesem Wettlauf keine echte Chance (IV, 327).

Im Verlauf des Romans muss Patap einige Rückschläge hinnehmen. Seine Investition in die Goldsuche an der Wetluga geht schief. Er trifft dort nicht auf Gold, sondern auf die Produktion von Falschgeld, und zwar durch altgläubige Geistliche. Nach seiner Heimkehr stirbt die älteste Tochter Nastja. Verführt von dem Aufsteiger und Luftikus Aleksej, den sich Patap selbst ins Haus geholt hatte, geht sie an ihrem Unglück zugrunde. Patap ist zutiefst bestürzt, aber er erholt sich, wie auch seine Ernährungsleistungen eher zu- denn abnehmen. Schon der Leichenschmaus gerät üppiger als die Namenstagsfeier zu Beginn des Romans. Für die сорочины Nastjas steigern sich Ausgaben und Kochkünste der Familie noch einmal. Gegen Ende des Romans kann Čapurin die zweite Tochter verheiraten. Das

9 Relativ klare Angaben bezuglich der Jahreszahl finden sich in II, 317 und IV, 264. In IV, 124 wird die laufende Handlung auf den 26. Juli datiert, danach allerdings feiert man Johannes sowie Peter und Paul, d. h. Feste, die in den Juni fallen. Die Chronologie ist hier aufgehoben, generell stehen in „V lesach" präzise Zeitangaben vorwiegend in Anmerkungen, die sich nur peripher auf die Handlung beziehen. Im Haupttext wird dagegen der Eindruck einer dauerhaften Lebensweise erzeugt, die dem Zeitverstăndnis des Epos entspricht. Levin 1958, 16 datiert die Handlungen von „V lesach“ und „Na gorach" auf die Jahre 1849 bis 1853 . Er bezieht sich jedoch auf die Chronologie historischer Ereignisse und nicht auf die Chronologie dieser Ereignisse im literarischen Text. 
Hochzeitsfest beschließt den Text. Es dauert ganze 11/2 Wochen, weil Patap nach einer Woche vom Feiern noch immer nicht genug hat.

„V lesach" ist kein Roman, der primär von der Handlung lebt und schon gar nicht von einer einzigen. Pataps Geschichte hat dennoch paradigmatische Bedeutung. Wie Čapurin arbeiten die meisten Figuren im Bereich von Handel und Gewerbe, während die Landwirtschaft eine ganz marginale Rolle spielt. Selten wird der Arbeitsprozess beschrieben, es zählt dagegen das Ergebnis, der Erfolg. Man erwirtschaftet Vermögen, das sich seinerseits in Nahrung niederschlägt. „In den Wäldern“ korrespondieren Kwas und Bier mit Vermögen, nicht notwendig Champagner mit Geld. Vor allem sind die ursprünglich russischen Getränke kein Indiz von Armut. Mel'nikovs Helden gehören weder der gesellschaftlichen Elite an - keine Aristokratie oder Intelligencija, die hier voll Mitleid auf die Bauern blickt -, noch zählen sie zu ,Erniedrigten und Beleidigten'. Den gesellschaftlichen Dualismus, von dessen emotionaler Spannung ein beträchtlicher Teil der russischen Literatur lebt, umgeht Mel'nikov elegant. Im Zentrum seines Romans steht die Kaufmannschaft, eine Mittelschicht, die sich primär aus sozial aufgestiegenen gewerbetreibenden Staatsbauern rekrutiert. ${ }^{11}$ Die Figuren sind im besten Alter, die Jüngsten heiratsfähig, auf der Suche nach Partnern, ihre Eltern: agil und geschäftstüchtig. Alte, Kranke und Kinder, die Gebrechlichen und Schutzlosen, kommen kaum vor. Und die Moral von der Geschicht' lässt sich wie folgt umschreiben: Man kann etwas erreichen - gesellschaftlich, ökonomisch, auch physisch -, wenn man nur will. An günstigen Bedingungen mangelt es hinter der Wolga jedenfalls nicht. Der Schlüssel zu Mel'nikovs Volkskonzept liegt in der Ökonomie, sie hat ihr Spiegelbild in Speisen und Getränken. Umgekehrt gesehen: Im Motiv der Nahrung verdichtet sich Mel'nikovs nationales Projekt, das auf die Entwicklung von einheimischem Kleingewerbe setzt und ohne soziale Spannungen auskommen will. Die ökonomische Philosophie bringt der designierte Schwiegersohn auf den Punkt: „Не в лесе [...] сила, а в промыслах [...] Будь по хлебным местам, как здесь, промысла, умирать бы не надо“" (III, 359).

Je unfruchtbarer die Erde, desto erfindungsreicher ihre Bewohner, desto vielfältiger und marktfreudiger ihre Produkte. Getreide hingegen erzielt nur einen Preis, der bei Überangebot, d.h. bei guter Ernte sinkt. Die Konsequenzen schlagen sich, wie Vasilij Borisyč pointiert formuliert, in der Suppe nieder: „По вашим местам - щи с наваром, крыты жиром, что их не видать, а в хлебных местах щи хоть кнутом хлещи - пузырь не вскочит..." (III, 358).

„V lesach" unterstützt weder die von staatlicher Seite geforderte народность, noch ein quasi ideales bäuerliches Leben. Vielmehr plädiert der Roman für den gemäßigten Aufbau eines bürgerlichen - besser gesagt kleinbürgerlichen - Milieus. Die erste Gemeinsamkeit der zutiefst verwobenen Bereiche von Essen und Kleingewerbe besteht im Variantenreichtum. Nicht Quantität oder Qualität einer einzelnen Speise sind von Bedeutung, und schon gar nicht zählt das rohe Material, etwa Getreide. Die кормы Čapurins zeichnen sich durch eine Vielzahl an Gerichten aus, durch das endlose Hintereinanderweg von Vorspeisen, ersten und zweiten Gängen sowie Desserts mitsamt einem reichen Angebot an Getränken. Mel'nikov listet Menüs auf, Speisekarten gleich, angesichts derer seinen Lesern

11 Auch Patap Maksimy飞 ist noch immer als Staatsbauer eingeschrieben (II, 47; IV, 356). 
das Wasser im Munde zusammenläuft. Die закуски einer einzigen Mahlzeit hören sich etwa wie folgt an:

Тут опять явились стерляди разварные с соленьми огурцами да морковыю, кроме того поставлены были осетрина холодная с хреном, да белужья тешка с квасом и капустой, тавранчук осетрий, щука под чесноком и хреном, нельма с солеными подновскими огурцами, а постнику грибы разварные с хреном, да тертий горох с ореховым маслом, да каша соковая с маковым маслом (II, 282).

Mit diesen appetitanregenden Listen bestätigt der Autor, was schon im Mittelalter als gutes russisches Essen galt. Geschirr, Besteck, das ganze Drumherum fällt in der Bedeutung weit hinter der Nahrung selbst zurück (Smith; Christian 1984, 116). Noch im 17. Jahrhundert beklagen sich ausländische Reisende über mangelnde russische Tischsitten, Katharina II. sucht dem Abhilfe zu schaffen, an ihrem Hof kommen kluge Tischgespräche in Mode, unter denen nun freilich die Küche leidet, die als schlechteste in Europa gilt (Munro 1997, 33f.). Mel'nikov verhilft den alten Werten wieder zu Rang. Wichtig ist, was gegessen und getrunken wird, - wie man sich dabei benimmt, kümmert den Russen wenig: Es muss eine große Auswahl herrschen. Der Bauer Evgraf steckt sich in Nižnij sogar noch die Reste einer Gans, eines Ferkels und Gurken in die Taschen, er fällt damit bei den Kellnern nicht aber bei den Gästen des Lokals auf, die gehobeneren Schichten angehören (III, 259f.). Die Orientierung auf Produkte und ihre möglichst große Anzahl trifft in „V lesach“ sowohl auf das Essen als auch auf das Gewerbe zu. Holzverarbeitende Betriebe, Spinnerei, Weberei, Fischfang, Hut- und Schuhmacherei flankieren zahlreiche Menüs mit auffallend vielen Fischgerichten.

Die bloße Konservierung von Traditionellem reicht jedoch für Wirtschaft und Fortschritt nicht aus. Das sieht der Autor, und er hat - wenngleich in begrenztem Maße - den Fortschritt im Sinn. „V lesach“ ist keine Apotheose dessen, was immer schon war und immer noch bleiben soll. In ökonomischer Hinsicht muss man riskieren, nicht allzu viel, aber doch ein bisschen, auch das Risiko manifestiert sich im Essen und besonders im Trinken. Die national-ökonomische Implikation der Getränke bringt beispielhaft eine Figur mit (ent-)sprechendem Namen zum Ausdruck. Samokvasov, ein windiger, im Grunde aber gutmütiger Bursche, macht seiner Familie alle Ehre, als es auch noch seinen (Vor-) Namenstag zu feiern gilt (IV, 216 ff.). Samokvasov ist nicht nur russisch, weil Kwas besonders russisch ist, er blickt auch auf eine entsprechende, ,selbstgebraute' Ahnengeschichte zurück. Sein Urgroßvater war ein zunächst eigenmächtig handelnder Räuber und Mörder, er agierte später unter Stepan Razin und hinterließ seiner Familie ein beträchtliches Vermögen, von dem Petr Stepanyč noch immer zehrt (IV, $136 \mathrm{ff}$.). Zwar fließt ein Teil des Geldes nun in die altgläubigen скиты, wo man für des Urgroßvaters sündige Seele betet, gleichzeitig investiert Samokvasov aber beachtliche Summen in die Entführung heiratswilliger Mädchen aus eben diesen скиты (IV, 324). Gegen Ende des Romans realisiert er, dass er das Erbe des Urgroßvaters nicht nur ausgeben, sondern in ein Unternehmen stecken sollte. Er will jenseits der Gebete und Schmiergelder sinnvoll investieren. Aber nicht von ungefähr denkt er über eine Beteiligung am Fischereigewerbe nach (IV, 285). Nahrung ist das Sicherste, das Einheimisch-Russische, Fische schwimmen in der Wolga zuhauf, und der wirtschaftliche Gewinn scheint garantiert. Samokvasov öffnet sich dem Neuen, potentiell sogar dem Ausländischen - markiert in seiner „deutschen“ Kleidung 
(IV, 126, 314) -, aber er bleibt im Kern russisch ${ }^{12}$. Auf das rechte Verhältnis und die rechte Verteilung kommt es hier an. Das Nationale, so will es „V lesach“, muss Grundlage des Handelns bleiben, im Kwas verdichtet sich eine Wirtschaftsphilosophie, die Tradition mit Risiko, Selbständigkeit mit Kollektiv und sogar Geld mit Lust verbindet. Anders dagegen steht es um den Schnaps.

Wenn heutzutage ein Klischee von ,dem Russen " existiert, so dürfte es sich um den Wodkatrinker handeln. Die nationale Natur des Wodka ist historisch gesehen aber fragwürdig. Als einheimisches, doch keineswegs traditionelles Getränk wird der Getreideschnaps weniger vom Volk als vom Staat favorisiert. Der Grund leuchtet ein: Das Monopol auf Brennereien und кабаки garantiert riesige Steuereinnahmen. Salopp formuliert: Das Volk muss saufen, damit der Fiskus verdient (Smith 1980, 50-52; Smith; Christian, 1984, 148, 288326). Diese staatlich organisierte Erniedrigung des Volkes, die in Verdummung und Verrohung gipfelt, machen die Menschen in „V lesach" nicht mit. Monopole jeder Art werden abgelehnt, explizit Fabriken, die nur deutsches Kapital vergrößern (III, 360), und implizit der Staat. Zwar serviert man reichlich Spirituosen, aber es handelt sich vorwiegend um Kräuterschnäpse, und diese kommen, das steht zu vermuten, aus privaten, illegalen Brennereien. So sabotiert der Alkoholkonsum ,in den Wäldern" auf heitere Weise den Staatsbetrieb. „V lesach“ plädiert für unternehmerische Eigeninitiative, selbst auf der Ebene des Schnapses. Nur ein einziger Säufer durchgeistert den Text, der Alkoholismus entfernt ihn aber von seiner wahren russischen Natur, seine Frau wäre, um ihn loszuwerden, sogar bereit einen Juden oder Tataren zu heiraten (II, 102). Der Mann wird von seiner Sucht geheilt, alle anderen Figuren trinken über den Durst zum Vergnügen und sind am nächsten Tag wieder nüchtern. Dabei präferiert man traditionelle russische Getränke, Bier, Braga, Met, Kwas, und geizt auch nicht mit importierten Waren. Čapurin offeriert Lafitte, ohne genau zu wissen, um welche Delikatesse es sich handelt (II, 168) ${ }^{13}$. Der Vorrat an ausländischen Weinen gehört zum Wohlstand, - sie zeichnen den russischen Tisch schon im Mittelalter aus - man bildet sich aber nichts darauf ein. Gesellschaftlich gesehen, erzeugt der Genuß von Lafitte keine Differenz zwischen Ober- und Unterschicht. Er kann auch dem echt russischen Menü nichts anhaben, und Mel'nikovs Held setzt sich hier betont von Onegins Dandyallüren ab.

Mehr als Lafitte bedeutet Čapurin dagegen der Tee. Hier liegt eine klare Neuerung des Speisezettels, eine echte Entsprechung zu ökonomischer Prosperität vor. Tee trinken heisst reich sein, zumindest in Russland Mitte des 19. Jahrhunderts (Smith; Christian 1984, 241). Für den Tee bricht Patap sogar mit traditionellen Speiseregeln. Er integriert das neue Getränk - unter Protest der Köchin - in ein altes Menü (II, 130) und beeindruckt mit dieser Kombination seine städtischen Gäste. Die Demonstration von Reichtum - Tee - auf der Basis von Tradition - den sogenannten заедки - gelingt. „In den Wäldern“, so könnte man Pataps Nahrungsbotschaft umschreiben, ist man noch lange kein Hinterwäldler. Der ausgiebige Teekonsum in „V lesach“ fällt auf, allerdings sind dabei soziale Abstufungen zu

12 Die menschliche Fassade lässt sich in „V lesach“ vom Ausland inspirieren, während das Innere - der Bauch - und auch die Fuße Widerstand zeigen. So muss der Hutmacher Zaplatin die Verschonerung von Köpfen seinen deutschen Konkurrenten uberlassen. Er verlegt sich klugerweise auf Bodenständigeres und produziert nunmehr валенки (II, 113-115).

13 Das Motiv wiederholt sich bei den Klosterbrudern: Angeblich versteht ihr Vorsteher nichts von fremden alkoholischen Getrănken, er serviert dennoch den besten Portwein (II, 282). 
vermerken. Bauern besitzen keinen Samowar und werden auch nicht mit Tee bewirtet. Dieser Mangel impliziert keine Armut - es gibt genug Ersatzgetränke -, außerdem lässt er sich beheben. Durch redliche Arbeit gelingt der soziale Aufstieg zum Tee, nicht jedoch zu Kaffee oder Schokolade. Und dafür gibt es Gründe.

Der echte russische Mann ist nämlich - die Leser werden darüber eigens belehrt - sauer, salzig, trinkfest, alles - nur nicht süß (III, 259). Süß muten hinter der Wolga allein die Frauen an, sie werden dementsprechend vernascht. In „V lesach" hat Nahrung außer der ökonomischen noch eine sexuelle Konnotation, die sich deutlich mit patriarchalischen Gesellschaftsstrukturen verbindet. Das nationale Modell des Autors erhält auf diese Art einen prekären Widerspruch. Frauen zeigen jenseits des Kochens keine unternehmerische Begabung, sie sind offenbar für jede Entscheidung zu blöde ${ }^{14}$. Süßes kann nicht bewusst und konstruktiv handeln. Das demonstriert gegen Ende des Romans sogar ein Mann, der Bauernsohn Aleksej. Er gerät im Verlauf des Textes immer mehr ins weibliche Fahrwasser. Seinen sozialen Aufstieg verdankt er der bedingungslosen Hingabe von Frauen, er erschleicht sich Vermögen, die Karriere mündet in Kaffee und Schokolade. „[...] самый интересный этот напиток "чиколат" [...]. Самый наилучший - а ла ваниль...“ (IV, 340) - mit diesem Getränk brüstet sich Aleksej vor Čapurin. Dem allzu süß gewordenen Bauern prognostiziert Patap jedoch wirtschaftlichen Ruin, und er kann hier mit dem Einverständnis des Lesers rechnen, denn das Bild von Aleksej ist negativ überzeichnet. ${ }^{15}$ Der Aufschneider und degenerierte Mann wird sein Kapital aufs schnellste durchbringen. Ökonomischer Erfolg - so suggeriert uns „V lesach“ braucht ,echte ' Männlichkeit. ${ }^{16}$

Wenn Frauen schon nicht wirtschaften können, so sind sie doch immerhin fruchtbar. „In den Wäldern" feiert man diese Fruchtbarkeit sogar intensiv. Mel'nikov greift dazu breit auf die Folklore zurück, er zitiert Lieder, Mythen, Rituale. Der alte slavische Gott Jarilo, Zeichen der Fruchtbarkeit, kommt zum Vorschein (III, 201ff.; IV, 84ff.), Kräuterweiblein werden angeworben, um den Geliebten zurückzubringen (III, 386ff.; IV, 16ff.), Frauen lösen die Gürtel für das Wachstum von Gurken, nackte Mädchen beschwören des Nachts ihre Gartenbeete (III, 303f.). Selten liegt die sexuelle Symbolik des Essens so klar auf der Hand wie in Mel'nikovs Roman. Der Produktivität des Bodens entspricht direkt und ohne Umschweife die physische Produktivität der Menschen. Wie wird man schwanger? „V lesach“ liefert quasi das Rezept: Man gehe Pilze suchen! Reihenweise werden Mädchen

14 Diese Unfahigkeit stellt besonders Mar'ja Gavrilovna zur Schau: Sie bezeichnet sich selbst als „dummes Weib" und gibt ihr gesamtes Kapital in die Hand des Geliebten Aleksej (IV, 28).

In Pochlebkins Studie zum Nahrungsrepertoire des russischen Dramas tritt Schokolade erst gegen Ende des 19. Jahrhunderts auf. (Pochlebkin 1993, 405) Die Verbreitung der Schokolade im Jahr 1852 scheint uberaus fragwürdig. Mel'nikovs polemisch uberzogene Konstruktion des Aufsteigers Aleksej kommt sowohl durch seinen Eingriff in die direkte Rede der Figur als auch durch der Wahl des Getränks zum Ausdruck. Zur Markierung luxuriösen Lebens hätten auch Kaffee und Tee gereicht, der Genuss von Schokolade geht über jeden Realitatsbezug hinaus und indiziert im Romangeschehen primär das Suße.

Der Fall Aleksej widerspricht Sombarts These, wonach ein kausaler Zusammenhang zwischen dem Süßen (Weiblichen) und der Entwicklung des Kapitalismus besteht (Sombart 1983, 61-134) In „V lesach" sind süße Frauen zumindest nicht Motor einer männlich dominierten Ökonomie. Das Illegale - etwa die Entführung von Mädchen und heimliche Hochzeiten - ist sogar billiger als die reguläre Variante und wird deshalb bevorzugt. Mănner investieren nicht in Frauen - „Kind der illegitimen Liebe" (ebenda, 194) ist also keineswegs der Luxus -, sondern sie sparen durch Frauen. (II, 72-75). 
beim Sammeln von Beeren und Pilzen verführt, uneheliche Kinder finden sich zuhauf, selbst die strenge игуменья Manefa lebt mit der Frucht ihrer Sünde im обитель. Die überaus heitere Konkurrenz von Kirche und weltlicher Leiblichkeit, die Mel'nikov im gesamten Text konstruiert, sei an einem Beispiel verdeutlicht. Eine alte Frau geht jährlich beichten, immer dasselbe: ,wie sie damals, als die Jahre noch golden und die Wangen noch rot waren, bei den Pilzen die Unschuld verlor'. Gott habe ihr längst verziehen, so der Priester, der Reue sei nun genug. Doch damit gibt sich die Alte eben nicht zufrieden: „„Батюшка, - отвечает старушка, - как же мне, грешнице, хоть еще разок не покаяться? Сладкое ведь сладко и вспомнить“"(III, 309).

Süß sind die Frauen, süß ihre Erinnerungen, aber nur auf Zucker und Honig will der Autor die Geschlechterbeziehungen nicht bauen. Nötig scheint vielmehr eine männliche Dominanz, die Herrschaft auch mit Gewalt verbindet, und so bedient sich Mel'nikov der entsprechenden Metaphorik, nämlich des Fleischfressens. ${ }^{17}$ Die carnivore Gesellschaft - so lautet die postmoderne Diagnose - ist eine phallogozentrische ${ }^{18}$, generell wird in der Forschung eine Affinität von Fleischkonsum und Machtstrukturen vermerkt ${ }^{19}$. Vegetarische Ernährung tendiert weniger zu sozialen Differenzen. Das liegt offenbar in der Natur der Sache. Zwischen Filet und Knochen besteht ein größerer Unterschied als zwischen Kraut und Rüben. ${ }^{20}$ Zwar kann man die Allgemeingültigkeit dieser These bezweifeln, und eklatante Ausnahmen - wie der Vegetarier Hitler - machen auch den Interpreten Mühe ( Adams 1990, 152; Derrida 1998, 428f., Anm. 18). Mel'nikovs „V lesach“ allerdings be stätigt die Regel: Schon die existierende, wenngleich nicht absolut gesetzte Schichtung der Gesellschaft kommt in der Fleischverteilung zum Ausdruck: Bauern essen vorwiegend Schweinefleisch (III, 147) ${ }^{21}$, für die Kaufleute dagegen ist Wild reserviert (III, 148). Zwischen den Klassen sind Übergänge noch vorgesehen - auf Čapurins Tisch findet sich hier und da auch ein Schinken (II, 130) -, zwischen den Geschlechtern keineswegs. Das Patriarchat bekundet sich im Fleischgenuss, der primär auf situativ-metaphorischer Ebene stattfindet. Junge Mädchen werden mit ungezähmten Kälbern verglichen (III, 277) oder gar anders herum: stattliche Kühe mit Gräfinnen aus Tambov (II, 284). Capurins autoritäre

17 Offensichtlich ist der Einsatz von Tier-, Fleisch- und Essmetaphern im Bereich der Pornographie. Umgekehrt nimmt die Fleischwerbung bisweilen den mănnlichen Blick auf weibliche Objekte zu Hilfe. Entsprechende Beispiele finden sich in Adams 1990, 58.

Derrida spricht von einem „Karno-Phallogozentrismus“, wobei es sich um eine Synthese a priori handele $(1998,291)$.

19 Canetti 1960, 231-255, geht dem Zusammenhang von Macht und Essen im allgemeinen nach, der Vergleich unterdruckter Menschen mit einverleibten Tieren (ebenda, 239) ist jedoch aufschlussreich. Den erotischen Aspekt des Kannibalismus demonstriert Brittnacher 1994, 69ff. LeBlanc 1997, 81-102, weist die Verbindung von Vegetarismus und sexueller Abstinenz bei Tolstoj nach und sieht in der carnivoren Metaphorik Dostoevskijs einen prägnanten Ausdruck des Machtverhältnisses zwischen den Figuren (LeBlanc 1997, 124-145).

Auch die jüdischen Ernăhrungsvorschriften, die zur nationalen Identifikation und zur Abgrenzung von Nicht-Juden fuhren, beziehen sich primär auf den Fleischkonsum.

21 Am deutlichsten wird die soziale Implikation in Capurins abwertendem Vergleich des Bauernsohnes Aleksej mit einem Schwein (IV, 338). 
Verheiratungsstrategie erhält die Etikette des Röstens (II, 24) ${ }^{22}$, männliches Begehren erscheint als Jagen, Schießen und Fressen (III, 309) ${ }^{23}$. So manches Täubchen wird zwar nur angeschossen (IV, 110), andere aber regelrecht verspeist. Das gilt vor allem für Nastja: Ihr Tod lässt sich zum einen als Fleischkonsum Aleksejs lesen - die Verführung bedeutet auch Vernichtung -, zum anderen als Fleischesopfer des Vaters ${ }^{24}$. Leibliche Hingabe, Sarg und Tisch gehen förmlich ineinander über. ${ }^{25}$ Werden Mädchen nicht eigens gefressen, so werden sie doch wenigstens geraubt. Und da der Raub zur Hochzeit führt, gerät die nachfolgende Ehe zum langsamen Verspeisen. Räuberisch zumindest erlangt Čapurin seine Aksin'ja, übermächtig wirkt seine Natur neben der dümmlichen Gattin. Aksin'ja gewinnt im gesamten Roman nicht das geringste Profil, selbst die Pseudobestrafung der neuvermählten Tochter überlässt sie am Ende ganz ihrem Mann. Sie hat nicht einmal die Pfannengabel in der Hand, die ihr laut Ritual für den Bestrafungsakt zustünde (II, 74). Während Patap ungeniert mit der Peitsche arbeitet, fuchtelt Aksin'ja nur mit den Armen (IV, 367).

Und damit stellt sich die Frage nach den Pfannen samt Inhalt, den блины. Verkörpern nun Pfannkuchen den Zusammenhalt der Gesellschaft, kommt ihnen gar eine nationale Funktion zu? Zunächst einmal sind блины in „V lesach“ Indiz sozialen Ausgleichs. Mit блины speist Čapurin das Volk, und die Bauern zeigen sich ihrerseits hilfsbereit. Man stellt 18 Öfen zur Verfügung und beteiligt sich am Backen, damit alle Gäste gleichzeitig das warme Produkt genießen können (III, 143, 147). Minimale Differenzen spiegeln sich im Gebrauch von Öl, hier wird analog zur Fleischverteilung zwischen besseren und einfachen Geladenen unterschieden (III, 326). Doch scheint die klassenspezifische Abstufung zweitrangig. Daneben aber und vor allem ist die Blinypfanne Indiz des Weiblichen, zu dessen genereller Charakteristik die Nahrungszubereitung in Gefäßen gehört (Lévi-Strauss 1997, 29-31). Über die Pfanne gebeugt gibt die Liebste den Blick in ihren Ausschnitt frei. Capurins Schwiegersohn erinnert sich wehmütig an dieses Bild mit seiner passenden Fortsetzung:

Блины пекла любушка Грушенька, а в келарне нас двое... Наклонится голубонька перед печкой, сковордник в руках... Стоишь рядом, заглянешь через плечи-то сверху под ворот... Искушение!.. Ну, известно, - тут бес... и ничего!.. Блинки поели и все позабыли... (IV, 119f.)

Pfannkuchen demonstrieren deshalb zweierlei: gesellschaftliche Homogenisierung und gesellschaftliche Differenz. Ökonomischer Wohlstand, der Bauern und Kaufleute zusam-

22 Lévi-Strauss 1997, 31, hebt die geschlechtsspezifische Bedeutung der Nahrungszubereitung hervor: Mit ,roasting“ wird das männliches Verhalten, mit ,boiling" das weibliche assoziiert. Inversionen sind möglich, Mel'nikov bestătigt jedoch das generelle Schema.

Das Fressmotiv wird nur in einem Falle umgekehrt, jedoch gelangt die resolute Ustin'ja nicht ans Ziel: Ihr erstrebtes Objekt, Vasilij Borisyč, hat sich anderweitig getröstet (III, 332, 335).

Das Opfer Nastjas fuhrt daruber hinaus zur Abstinenz des Saufers Nikifor und heilt ihn von seinem „wölfischen Geschăft“, dem Diebstahl (II, 103; III, 146). Es scheint als habe der „Wolf“ an dieser unerwarteten Beute genug. Aleksej dagegen verdirbt sich an Nastja quasi den Magen und gerăt auf die falsche Bahn.

Nastjas aufgebahrte Leiche wird mit Roggenăhren und duftenden Beeren geschmuckt, sie umrahmen den toten Leib wie Beilagen ein Fleischgericht (III, 138f.). Beim Blick auf den Tisch in Nastjas Raumen evoziert Aleksej den sterbenden Körper seiner Geliebten (III, 159). 
menfuihrt, konkurriert mit einem markanten Unterschied zwischen Geschlechtern. Männer jagen, Männer speisen, Frauen kochen und werden verspeist. Mel'nikovs Roman kombiniert quasi zwei Zeitalter miteinander: das nationale und das feudale, mit seinen herrschenden Instanzen von Familie und Patriarchat, die sich der Nationsbildung jedoch widersetzen (Hroch 1985, 152f.). Diesem Umstand dürften auch Stilbrüche und Gattungsmischungen des Textes zu verdanken sein. Mel'nikov steht - unentschieden - zwischen alt und neu, in historischer, literarischer, inhaltlicher und formaler Hinsicht. Die Idylle geht mit wissenschaftlichen Anmerkungen geschichtlicher, mythologischer und sogar botanischer Art einher. Realistische, nahezu dokumentarisch genaue Beschreibungen - ein Beispiel ist die Behausung von Holzfällern (II, $212 \mathrm{f}$.) - wechseln sich mit märchenhaften und folkloristischen Passagen ab. Letztere beinhalten die Geschlechterbeziehungen und sind keineswegs der russischen Wirklichkeit von 1852 entnommen. Dieses Volksleben hat Mel'nikov - wie Vinogradov deutlich nachweist - aus Büchern, genauer: aus den Sammlungen von Afanas'ev (Vinogradov 1936, 23ff.). Althergebrachtes, Mythologisches, das sich im übrigen nicht auf den Wolgaraum bezieht, geht ohne Korrektur in den neuen literarischen Text ein. Der Autor, der in Fragen der Ökonomie durchaus Tradition mit Innovation verbindet, bleibt für die Emanzipation der Frau ohne Gespür und Gehör. Selbst die Religion ist in Mel'nikovs Fortschrittsglauben einbezogen, die Raskol'niki erscheinen in „V lesach" überaus flexibel. So denkt man in Manefas обитель gar über die Zubereitung einer Kartoffelsuppe nach (IV, 157). Die Aversion gegen den vermeintlichen Paradiesapfel (Smith; Christian 1984, 200, 282) ignoriert Mel'nikov kurzerhand. Allzu starre, religiös motivierte Positionen werden aufgeweicht, einen Wandel des Geschlechterverhältnisses sieht „V lesach“ aber nicht vor. Das Volk ,in den Wäldern“ ist so gesehen ein gespaltenes, jedoch verläuft der Bruch nicht dort, wo man ihn im Horizont realistischer Literatur vermuten würde.

Es scheint zumindest ein Verdienst Mel'nikovs, einmal nicht die russische Seele zu bemühen, um den gebeutelten Körper des Volkes zu legitimieren. Mel'nikov beschwört den materiellen Wohlstand, den Bauch, das Essen und Trinken. Er setzt sich klar von Positionen ab, die magere bäuerliche Diäten zum Ideal einer gesunden Ernährung stilisieren ${ }^{26}$ und damit möglicherweise den Mangel festschreiben. In „V lesach“ essen die Bauern Fleisch - das sollen sie wohl auch. Vereinfacht gesagt: Mel'nikov will die ökonomische Situation des Volkes bessern, nicht voll Ehrfurcht auf arme Menschen blicken, die von Ehrfurcht und Mitleid am allerwenigsten haben. Darin hält er sich an Feuerbachs Thesen und darin liegt ein Vorzug.

\section{Literatur}

ADAMS, C. J., The Sexual Politics of Meat: A Feminist Vegetarian Critical Theory, New York 1990.

BRITTNACHER, H. R., Verzehrende Leidenschaft: Über Metaphern der Einverleibung, in: Verschlemmte Welt: Essen und Trinken historisch-anthropologisch, hrsg von A. SCHULLER und J. A. KLEBER, Göttingen; Zürich 1994, S. 64-88.

26 Dazu gehört der Chemiker Éngelgardt, der von 1871 bis 1887 Reflexionen uber eine besonders ,rationale' Ernăhrung der Bauern in den „Otexestvennye zapiski“ publiziert. Sowohl Frierson 1997, 61, als auch Smith; Christian 1984, 327f., machen jedoch darauf aufmerksam, dass Ėngelgardt bisweilen der Zusammenhang von kärglicher Nahrung und Ausbeutung entgehe. 
CANETTI, E., Masse und Macht, Hamburg 1960.

DERRIDA, J., Man muß wohl essen, in: DERS., Auslassungspunkte: Gespräche, Wien 1998, S. 267-298.

DOUGLAS, M., Deciphering a Meal, in: Food and Culture. A Reader, hrsg. von C. COUNIHAN und P. VAN ESTERIK, New York; London 1997, S. 36-54.

FEUERBACH, L., Die Naturwissenschaft und die Revolution: (Über: Lehre der Nahrungsmittel. Für das Volk. Von Moleschott), in: DERS., Gesammelte Werke, Bd. 10: Kleinere Schriften III (1846-1850), Berlin 1971.

FRIERSON, C. A., Forced Hunger and Rational Restraint in the Russian Peasant Diet: One Populist's Vision, in: Food in Russian Literature and Culture, hrsg. von M. GLANTS und J. TOOMRE, Bloomington 1997, S. 49-66.

HARRIS, M., The Abominable Pig, in. Food and Culture. A Reader, a. a. O, S. 67-79.

HERETZ, L., The Practice and Significance of Fasting in Russian Peasant Culture at the Turn of the Century, in: Food in Russian Literature and Culture, a. a. O., S. 67-80.

HOBSBAWM, E. J., Nationen und Nationalismus: Mythos und Realitat seit 1780, Frankfurt/Main; New York 1991.

HOSKING, G., The Russian National Myth Repudiated, in: Myths and Nationhood, hrsg. von DEMS. und G. SCHOEPFLIN, London 1997, S.198-210.

HOSKING, G., Russischer Nationalismus vor 1914 und heute: Die Spannung zwischen imperialem und ethnischem Bewußtsein, in: Die Russen: ihr Nationalbewusstsein in Geschichte und Gegenwart, hrsg. von A. KAPPELER, Koln 1990, S. 169-183.

HROCH, M., Social Preconditions of National Revival in Europe, Cambridge 1985.

KAPPELER, A., Bemerkungen zur Nationsbildung der Russen, in: Die Russen: ihr Nationalbewusstsein in Geschichte und Gegenwart, a. a. O., S. 19-35.

KAPPELER, A., Russland als Vielvolkerreich, München 1992.

KNIGHT, N., Ethnicity, Nationality and the Masses: Narodnost' and Modernity in Imperial Russia, in: Russian Modernity: Politics, Knowledge, Power, hrsg. von D. L. HOFFMANN und Y. KOTSONIS, Basingstoke; London 2000, S. 41-64.

LEBlanC, R. D., An Appetite for Power: Predators, Carnivores, and Cannibals in Dostoevsky's Fiction, in: Food in Russian History and Culture, a. a. O., S. 124-145.

LEBLANC, R. D., Tolstoy's Way of No Flesh: Abstinence, Vegetarianism, and Christian Physiology, in: Food in Russian History and Culture, a. a. O., S. 81-102.

LEvIN, F., Epopeja P. I. Mel'nikova (Andreja Pecerskogo), in: P. I. MEL'NIKOV, Na gorach, Bd. 1, M. 1958. S. 3-28.

LÉVI-STRAUSS, C., The Culinary Triangle, in: Food and Culture. A Reader, a. a. O., S.28-35.

MEL'NIKOV, P. I. (Andrej Peðerskij), V lesach, in: DERS., Sobranie socinenij v vos'mi tomach, Bde. 2-4, M. 1976.

MUNRO, G. E., Food in Catherinian St. Petersburg, in: Food in Russian History and Culture, a. a. O., S. 31-48.

POCHLEBKIN, V. V., KuŠat' podano: Repertuar kušanij i napitkov v russkoj klassiceskoj dramaturgii s konca XVIII do nacala XX stoletija, M. 1993.

SOMBART, W., Liebe, Luxus und Kapitalismus: Über die Entstehung der modernen Welt aus dem Geist der Verschwendung (1913), Berlin 1983.

SMITH, R. E. F., Drink in Old Russia, in: Peasants in History, hrsg. von E. J. HOBSBAWM u. a., Oxford 1980. S. $42-54$.

SMITH, R. E. F.; CHRISTIAN, D., Bread and Salt: A Social and Economic History of Food and Drink in Russia, Cambridge 1984.

SOLER, J., The Semiotics of Food in the Bible, in: Food and Culture. A Reader, a. a. O., S. 55-66.

VINOGRADOV, G. S., Fol'klomye istočniki romana Mel'nikova-Pecerskogo „V lesach“, in: P. I. MEL'NIKOV (Andrej Pecerskij), V lesach, Bd. 1, M.; L. 1936. S. 8-67.

Dr. Andrea Zink, Slavisches Seminar, Universität Basel, Nadelberg 4, 4051 Basel, Schweiz (Andrea.Zink@unibas.ch) 